\title{
The Cognitive Correlates of Human Brain Oscillations
}

\author{
Michael J. Kahana \\ Department of Psychology, University of Pennsylvania, Philadelphia, Pennsylvania 19104
}

This mini-symposium summarizes recent findings concerning the role of brain oscillations in human cognition. Two rhythms that show particularly strong behavioral correlates are the $4-8$ $\mathrm{Hz}$ theta rhythm and the $30-80 \mathrm{~Hz}$ gamma rhythm. The former rhythm has been studied extensively in rodents, in which it is especially prominent in the hippocampus during locomotion, orienting, and other voluntary behaviors. The higher-frequency gamma rhythm has been linked recently to increased hemodynamic activity (as measured using functional magnetic resonance imaging) in cat, monkey, and human. Recent analyses of intracranial electroencephalographic (iEEG) recordings, taken from neurosurgical patients, have provided evidence for locally generated brain oscillations in human hippocampus and neocortex. Along with scalp EEG and magnetoencephalographic (MEG) recordings, these studies reveal evidence of synchronous oscillations in the theta- and gamma-frequency bands that are modulated by behavioral and task variables. The convergence of recent evidence suggests that brain oscillations are generated in almost every part of the brain and that they play a wide range of functions in both human and animal cognition.

\section{Bridging between single units and EEG}

The time-varying voltage generated by ensembles of neurons can be measured at many different spatial scales, with extracellular recordings reflecting the activity of a few nearby cells and scalprecorded EEG or MEG signals reflecting the summed activity of many millions of neurons distributed over large regions of human cortex. Analyses of brain activity at these diverse spatial scales reveal distinct views of the behavior of the nervous system. Whereas analyses of single-cell recordings from visual or auditory cortex can show how these cells code for properties of a sensory input, analyses of scalp-recorded EEG or MEG signals can help to elucidate the timing of coherent neural activity throughout the entire visual or auditory cortex.

The neurophysiology of animal cognition has been primarily informed by recordings taken from individual cells or small populations of cells. In contrast, the electrophysiology of human cognition has been studied primarily using noninvasive measures of scalp-recorded EEG and MEG signals. Until very recently, a seemingly insurmountable gap separated the two levels of physiological measurements: at one level, animal studies, which used field potential and single-unit recordings, and at the other level,

Received Sept. 3, 2005; revised Dec. 2, 2005; accepted Dec. 17, 2005.

This research was funded by National Institutes of Health Grants MH55687 and MH61975. I am grateful to Per Sederberg, Marieke van Vugt, and Josh Jacobs for helpful comments and discussions.

Correspondence should be addressed to Michael J. Kahana at the above address. E-mail: kahana@psych.upenn.edu.

D01:10.1523/JNEUROSCI.3737-05c.2006

Copyright $\odot 2006$ Society for Neuroscience $\quad$ 0270-6474/06/261669-04\$15.00/0 human studies, which focused on subtle changes in the scalprecorded EEG or MEG that result from the modulation of largescale, synchronous, neocortical oscillations.

Recent work has attempted to bridge these different levels by recording neural activity from electrodes implanted inside the human brain as part of neurosurgical treatment of pharmacologically resistant epilepsy. Every year, $\sim 1500$ epileptic patients in the United States undergo invasive iEEG monitoring with subdural grids and/or depth electrodes to help localize their seizure foci for subsequent resection. After resection of their focus, approximately half of these patients have significant reduction of seizure frequency. Because the clinical procedure of locating seizure foci requires electrode placement on regions only hypothesized to be epileptogenic, there are typically far more electrodes covering nonepileptogenic areas than areas that will eventually prove to be the focus. The high signal-to-noise ratio characteristic of iEEG recordings helps to locate the areas of steep voltage gradients indicative of signal sources in the human brain.

Although data from iEEG recordings have been used clinically for $>50$ years, it is only recently that the method has been used together with controlled experiments to characterize the electrophysiology of various cognitive functions (Fernandez et al., 1999; Kahana et al., 1999, 2001; Fell et al., 2001; Raghavachari et al., 2001; Ekstrom et al., 2003; Howard et al., 2003; Rizzuto et al., 2003; Sederberg et al., 2003; Tallon-Baudry et al., 2004; Lachaux et al., 2005). In these studies, recordings are taken from subdural electrode grids placed on the cortical surface, depth electrodes implanted within human medial temporal-lobe structures, or occasionally from microwires designed to record responses of individual cells (Krieman et al., 2000; Cameron et al., 2001; Fried et al., 2002; Ekstrom et al., 2003; Engel et al., 2004). A unique feature of recordings taken during surgical treatment of epilepsy is that the electrodes are kept in place over a period spanning many days, during which time patients are alert and capable of performing complex cognitive tasks. These long periods afford many opportunities to collect behavioral data from subjects who are not heavily sedated, as is the case with recordings taken in the operating room.

\section{Spectral analyses}

EEG data form a series of voltage time series measured from different locations in the human brain or on the scalp. Spectral analysis methods may be used to measure both the presence of oscillations within a single time series as well as the phase locking of oscillations across two different voltage time series. Spectral analysis of EEG data reveals two important features of the time series of EEG data. First, EEG data, like most natural processes, has more energy at low than at high frequencies (Fig. 1, left). This is a consequence of the autocorrelation within the time series; 

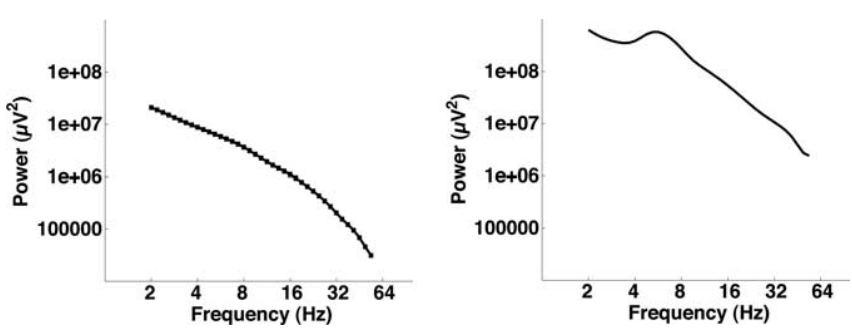

Figure 1. Power spectra of iEEG signals. Left, Average power spectrum calculated across 1808 intracranial electrodes in 35 epileptic patients. iEEG data were collected while patients studied and attempted to recall lists of common words. Right, Power spectrum from an electrode in Brodmann area 37 (near the fusiform gyrus) showing a peak in the $4-8 \mathrm{~Hz}$ theta frequency range. P. B. Sederberg, A. Schulze-Bonhage, J. R. Madsen, E. B. Bromfield, D. C. McCarthy, A. Brandt, M. S. Tully, M. J. Kahana, unpublished observation).

that is, the voltage at time $t$ depends on the voltage at time $t-1$. The second feature of the time series is the presence of increased amplitude (or power) at specific frequencies. This can be seen in data from a sample electrode that show a peak in the $4-8 \mathrm{~Hz}$ theta band (Fig. 1, right). The presence of peaks at specific frequencies implies that, in addition to its generally slow-changing voltage pattern, the EEG time series can also contain oscillations at specific frequencies.

\section{Electrophysiology of verbal and spatial cognition}

Oscillations in the theta-frequency range (usually defined as $4-8$ $\mathrm{Hz}$ ) have long been studied in animals in which they appear prominently in field recordings from the rat hippocampus. In rodents, theta oscillations, which increase during locomotion, orienting, conditioning, and maintenance of goal states, have played a crucial role in theories of the neural basis of animal learning and memory.

Motivated by these animals studies, Kahana et al. (1999) had patients with subdural grid electrodes learn to navigate through virtual, three-dimensional rendered mazes. Their recordings revealed clear oscillations in the unfiltered iEEG traces and demonstrated that, during maze navigation, intermittent bouts of theta activity appear with greater probability during longer mazes, even when controlling for degree of mastery. Caplan et al. (2001) showed that the effect of maze length on theta does not reflect the increased difficulty of encoding or retrieval at individual choice points. Rather, it reflects a global difference between long and short mazes. Caplan et al. found that gamma activity, but not theta, increased with increasing difficulty of individual choices at maze junctions. It was at these difficult junctions in which the learning requirements would have been greatest.

Because subjects often learn T-mazes as a verbal sequence of left and right turns (Kirschen et al., 2000), it is difficult to make strong inferences about the role of theta in spatial processing from Kahana and Caplan's earlier studies. To help address this confound, we developed a task called "Yellow Cab" in which subjects play the role of a taxi driver, driving through a virtual town in search of passengers and delivering those passengers to their requested destinations (Fig. 2). Over repeated deliveries, passengers learn to find the shortest path between the random locations in which they find their passengers and the fixed locations of landmarks within the environment (Caplan et al., 2003; Newman et al., 2005).

Caplan et al. (2003) found increased theta activity at widespread cortical sites when subjects were moving (compared with periods in which they were still). Extending these results by comparing iEEG recordings from hippocampus and neocortex, Ek-
A

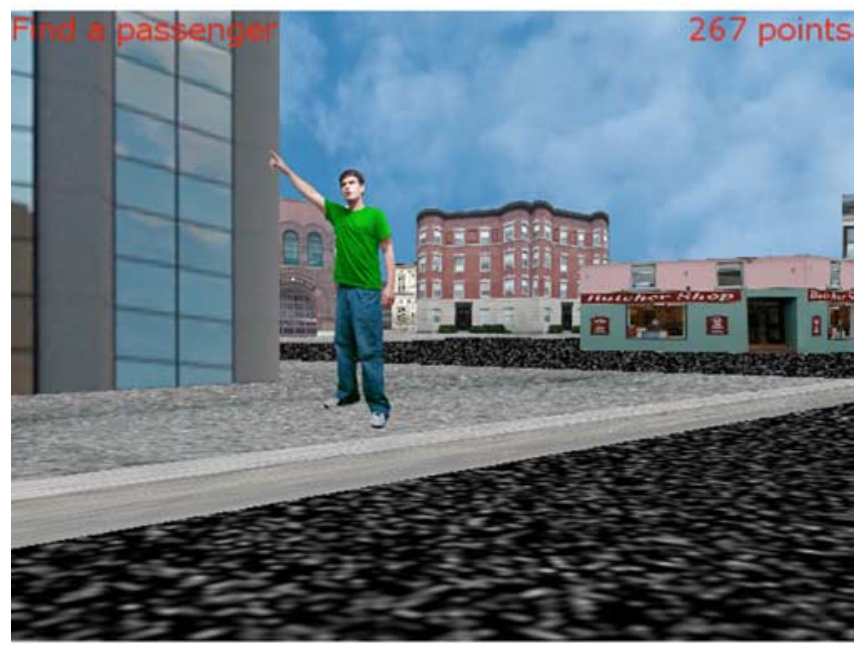

B

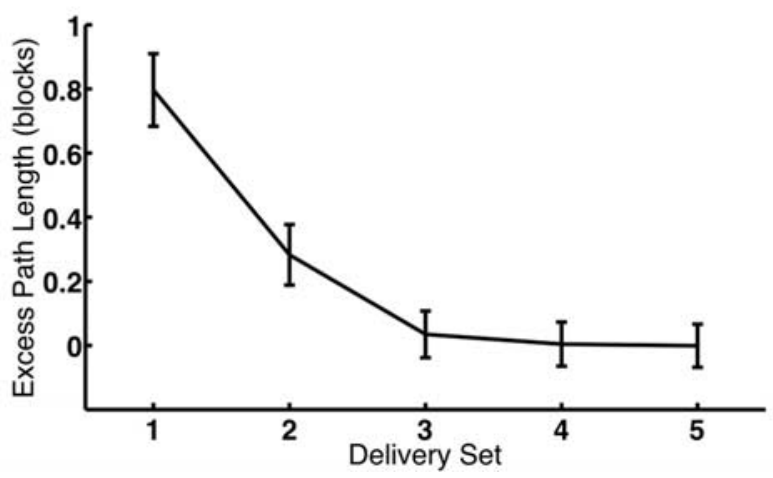

Figure 2. Yellow Cab: a virtual taxi-driver game. $\boldsymbol{A}$, Subjects drive a taxi through a virtual town seeking passengers (boy in green) and delivering them to target locations (stores). $\boldsymbol{B}$, Performance, measured as the difference between the path taken and the optimal path (excess path length), decreases with each delivery. Adapted from Newman et al. (2005).

strom et al. (2005) found increased theta activity in both regions when subjects were moving within the virtual town.

Analyzing recordings of neurons in hippocampal, parahippocampal, and frontal regions while patients played Yellow Cab, Ekstrom et al. (2003) found cells in the hippocampus that responded at specific spatial locations and cells in the parahippocampal region responding to views of landmarks. Cells also responded to the subject's navigational goals and to conjunctions of place, goal, and view. Work is currently underway to examine the phase locking of single-cell responses with the locally generated theta rhythm. Overall, these electrophysiological studies reveal a striking parallel between the neural basis of navigation in human and animals. We next turn to electrophysiological analyses of verbal memory tasks.

In humans, scalp EEG and MEG recordings, as well as iEEG recordings, have revealed increases in theta activity during verbal working memory tasks (Gevins et al., 1997; Raghavachari et al., 2001; Jensen and Tesche, 2002). Human cortical theta activity has also been shown to reset after both stimulus encoding and retrieval in a working memory task (Rizzuto et al., 2003), a result that parallels similar findings in the rat (Givens, 1996). Coherence analyses have shown significant long-range theta-band coherence between prefrontal and posterior electrodes during the 
retention interval of both a verbal and a visuospatial working memory task but not during a perceptual control task (Sarntheim et al., 1998).

In longer-term episodic memory tasks, both theta power and synchronization have been shown to increase during successful memory encoding (Klimesch et al., 1996; Weiss and Rappelsberger, 2000; Sederberg et al., 2003) and during recognition of previously studied items (Klimesch et al., 2006). This latter effect is typically seen in the left-parietal cortex and has been shown to be distinct from the left-parietal old-new effect seen in eventrelated potential studies (Klimesch et al., 2000). A similar leftparietal theta effect was reported by Bastiaansen et al. (2005) in the 300-500 ms after viewing open-class words (nouns, verbs, or adjectives) compared with closed-class words (determiners, conjunctions, or prepositions). The same study has shown that theta also correlates with word length. Overall, these studies show how theta correlates with the cognitive processing involved in encoding and retrieving verbal stimuli. Thus, one cannot link theta specifically to spatial processing.

Gamma-frequency oscillations $(30-80 \mathrm{~Hz})$ have also been implicated in a variety of verbal and nonverbal cognitive tasks. Gamma activity has been most widely associated with top-down attentional processing and object perception (Tiitinen et al., 1993; Keil et al., 1999; Rodriguez et al., 1999; Debener et al., 2003; Gruber and Muller, 2005), but it has also been shown to increase during a delayed match-to-sample task with visual stimuli (Tallon-Baudry et al., 1998, 1999, 2005) and with memory load in a working memory task (Howard et al., 2003).

Recording iEEG signals from the human medial temporallobe structures, Fell et al. (2001) reported that increased gamma synchrony between the rhinal cortex and hippocampus during the encoding period of a word predicted whether or not that word would later be recalled. Also analyzing iEEG recordings from widespread cortical sites, Sederberg et al. (2003) reported that increased gamma power during encoding predicted subsequent recall.

\section{Synthesis}

The past few years have seen increased interest in human brain oscillations and their possible role in perceptual and cognitive processes. Two oscillations that have received extensive attention are the theta rhythm $(4-8 \mathrm{~Hz})$ and the gamma rhythm $(30-80$ $\mathrm{Hz}$ ). This work comes on the heels of extensive animal work investigating the behavioral and physiological correlates of brain rhythms.

In our view, several factors are responsible for the surge of interest in human brain rhythms. First, mathematical methods used to study oscillations are far more accessible than before. Standard computer software now makes spectral analysis relatively easy, and modern computers have the necessary speed and memory capacity to apply these techniques to large datasets. Second, computational neuroscientists have pushed the experimentalists studying neurophysiology to consider network phenomena rather than focusing their attention primarily on the response recorded from a single electrode. Third, the ability to record EEG from small regions of the human brain using intracranial methods has demonstrated that prominent oscillations like those long studied in rodents and cats are also present in human cortex and hippocampus (Ekstrom et al., 2005; Raghavachari et al., 2005). Fourth, several theorists have hypothesized that the evoked potentials observed in a variety of cognitive and perceptual tasks may in fact reflect the reset of phase shifting of oscillations. Recent support for these ideas comes from demonstrations of stimulus-induced phase resetting of EEG reported by Makeig et al. (2002) and Rizzuto et al. (2003).

An extensive database is now emerging on the oscillatory correlates of a variety of cognitive processes. Whereas early efforts hoped to link a given oscillatory frequency with a specific cognitive process, the range of findings in the literature shows that these hopes have gone unfulfilled (Buzsaki et al., 2002; Bastiaansen and Hagoort, 2003). Instead, what has emerged is a rich picture of brain dynamics in which locally generated oscillations primarily in the theta and gamma bands, but also at other frequencies, appear in different brain regions and appear to be modulated by different cognitive processes. For example, theta may correlate with one behavior in a given brain region and poststimulus latency, but theta activity may correlate with another behavior at a different brain region or at a different poststimulus latency. This implies that, to progress, we need to use multivariate statistical methods that allow one to correlate a set of behavioral and task variables with a set of electrophysiological variables (including the time course of spectral power and the covariance between power and phase across recording sites).

\section{References}

Bastiaansen M, Hagoort P (2003) Event-induced theta responses as a window on the dynamics of memory. Cortex 39:967-992.

Bastiaansen M, van der Linden M, ter Keurs M, Dijkstra T, Hagoort P (2005) Theta responses are involved in lexical-semantic retrieval during language processing. J Cogn Neurosci 17:530-541.

Buzsaki G (2002) Theta oscillations in the hippocampus. Neuron 33:325-340.

Cameron KA, Yashar S, Wilson CL, Fried I (2001) Human hippocampal neurons predict how well word pairs will be remembered. Neuron 30:289-298.

Caplan JB, Madsen JR, Raghavachari S, Kahana MJ (2001) Distinct patterns of brain oscillations underlie two basic parameters of human maze learning. J Neurophysiol 86:368-380.

Caplan JB, Madsen JR, Schulze-Bonhage A, Aschenbrenner-Scheibe R, Newman EL, Kahana MJ (2003) Human theta oscillations related to sensorimotor integration and spatial learning. J Neurosci 23:4726-4736.

Debener S, Herrmann CS, Kranczioch C, Gembris D, Engel AK (2003) Topdown attentional processing enhances auditory evoked gamma band activity. NeuroReport 14:683-686.

Ekstrom A, Kahana MJ, Caplan JB, Fields TA, Isham EA, Newman EL, Fried I (2003) Cellular networks underlying human spatial navigation. Nature 425:184-187.

Ekstrom A, Caplan J, Ho E, Shattuck K, Fried I, Kahana M (2005) Human hippocampal theta activity during virtual navigation. Hippocampus 15:881-889.

Fell J, Klaver P, Lehnertz K, Grunwald T, Schaller C, Elger CE, Fernandez G (2001) Human memory formation is accompanied by rhinalhippocampal coupling and decoupling. Nat Neurosci 4:1259-1264.

Fernandez G, Effern A, Grunwald T, Pezer N, Lehnertz K, Dumpelmann M, van Roost D, Elger CE (1999) Real-time tracking of memory formation in the human rhinal cortex and hippocampus. Science 285:1582-1585.

Fries P, Schroder J-H, Roelfsema PR, Singer W, Engel AK (2002) Oscillatory neuronal synchronization in primary visual cortex as a correlate of stimulus selection. J Neurosci 22:3739-3754.

Gevins A, Smith ME, McEvoy L, Yu D (1997) High-resolution EEG mapping of cortical activation related to working memory: effects of task difficulty, type of processing, and practice. Cereb Cortex 7:374-385.

Givens B (1996) Stimulus-evoked resetting of the dentate theta rhythm: relation to working memory. NeuroReport 8:159-163.

Gruber T, Matthias M (2005) Oscillatory brain activity dissociates between associative stimulus content in a repetition priming task in the human EEG. Cereb Cortex 15:109-116.

Howard MW, Rizzuto DS, Caplan JB, Madsen JR, Lisman J, AschenbrennerScheibe R, Schulze-Bonhage A, Kahana MJ (2003) Gamma oscillations correlate with working memory load in humans. Cereb Cortex 13:1369-1374.

Jensen O, Tesche CD (2002) Frontal theta activity in humans increases with memory load in a working memory task. Eur J Neurosci 15:1395-1399. 
Kahana MJ, Sekuler R, Caplan JB, Kirschen M, Madsen JR (1999) Human theta oscillations exhibit task dependence during virtual maze navigation. Nature 399:781-784.

Kahana MJ, Seelig D, Madsen JR (2001) Theta returns. Curr Opin Neurobiol 11:739-744.

Kirschen MP, Kahana MJ, Sekuler R, Burack B (2000) Optic flow helps humans learn to navigate through synthetic environments. Perception 29:801-818.

Klimesch W, Doppelmayr M, Russegger H, Pachinger T (1996) Theta band power in the human scalp EEG and the encoding of new information. NeuroReport 7:1235-1240.

Klimesch W, Doppelmayr M, Schwaiger J, Winkler T, Gruber W (2000) Theta oscillations and the ERP old/new effect: independent phenomena? Clin Neurophysiol 111:781-793.

Klimesch W, Hanslmayr S, Sauseng P, Gruber W, Brozinsky CJ, Kroll NEA, Yonelinas AP, Doppelmayr M (2006) Oscillatory EEG correlates of episodic trace decay. Cereb Cortex 16:280-290.

Kreiman G, Koch C, Fried I (2000) Imagery neurons in the human brain. Nature 408:357-360.

Makeig S, Westerfield M, Jung T-P, Enghoff S, Townsend J, Courchesne E, Sejnowski TJ (2002) Dynamic brain sources of visual evoked responses. Science 295:690-694.

Newman EL, Caplan JB, Kirschen MP, Korolev IO, Sekuler R, Kahana MJ (2006) Learning your way around town: virtual taxi drivers reveal the secrets of navigational learning. Cognition, in press.

Raghavachari S, Rizzuto DS, Caplan JB, Kirschen MP, Bourgeois B, Madsen JR, Lisman JE (2001) Gating of human theta oscillations by a working memory task. J Neurosci 21:3175-3183.

Raghavachari S, Lisman JE, Tully M, Madsen JR, Bromfield EB, Kahana MJ
(2006) Theta oscillations in human cortex during a working memory task: evidence for local generators. J Neurophysiol, in press.

Rizzuto DS, Madsen JR, Bromfield EB, Schulze-Bonhage A, Seelig D, Aschenbrenner-Scheibe R, Kahana MJ (2003) Reset of human neocortical oscillations during a working memory task. Proc Natl Acad Sci USA 100:7931-7936.

Rodriguez E, George N, Lachaux JP, Martinerie J, Renault B, Varela FJ (1999) Perception's shadow: long-distance synchronization of human brain activity. Nature 397:430-433.

Sarnthein J, Petsche H, Rappelsberger P, Shaw GL, von Stein A (1998) Synchronization between prefrontal and posterior association cortex during human working memory. Proc Natl Acad Sci USA 95:7092-7096.

Sederberg PB, Kahana MJ, Howard MW, Donner EJ, Madsen JR (2003) Theta and gamma oscillations during encoding predict subsequent recall. J Neurosci 23:10809-10814.

Tallon-Baudry C, Bertrand O, Peronnet F, Pernier J (1998) Induced gamma-band activity during the delay of a visual short-term memory task in humans. J Neurosci 18:4244-4254.

Tallon-Baudry C, Kreiter A, Bertrand O (1999) Sustained and transient oscillatory responses in the gamma and beta bands in a visual short-term memory task in humans. Vis Neurosci 16:449-459.

Tallon-Baudry C, Bertrand O, Henaff M-A, Isnard J, Fischer C (2005) Attention modulates gamma-band oscillations differently in the human lateral occipital cortex and fusiform gyrus. Cereb Cortex 15:654-662.

Tiitinen H, Sinkkonen J, Reinikainen K, Alho K, Lavikainen J, Naatanen R (1993) Selective attention enhances the auditory $40-\mathrm{Hz}$ transient response in humans. Nature 364:59-60.

Weiss S, Rappelsberger P (2000) Long-range EEG synchronization during word encoding correlates with successful memory performance. Cogn Brain Res 9:299-312. 